\title{
Relation Entre L'activité Biologique Et Le Comportement Hydro Physique Des Sols Dans Le Contexte Du Systéme Prairial Naturel Irrigué A L'eau Usée
}

\author{
Ababsa Nawal \\ Université Ferhat Abbes, SETIF, ALGERIE \\ Université Abbes Laghrour, KHENCHELA, ALGERIE \\ Kribaa Mohammed \\ Université Ferhat Abbes, SETIF, ALGERIE \\ Tamrabet Lahbib \\ Laboratoire de recherche RNAMS, \\ Université LBM Oum El Bouaghi, ALGERIE

\section{Addad Dalila} \\ Université Abbes Laghrour, KHENCHELA, ALGERIE \\ Zidi Anissa \\ Mansouri Djawhara \\ Laboratoire de recherche RNAMS, \\ Université LBM Oum El Bouaghi, ALGERIE
}

\section{doi: 10.19044/esj.2016.v12n5p76 URL:http://dx.doi.org/10.19044/esj.2016.v12n5p76}

\begin{abstract}
The practice of irrigation using wastewaters is a promising solution to alleviate the problem of water stress in semi-arid regions. Our study focuses on the impact of the practice of irrigation with wastewaters on both physical and biological components of soil; as well as the interactive effects of these two components on water functioning in soil of eastern Algeria. The water analysis showed high organic and fine-particle pollutions, but with no significant influence on soil properties. Our findings revealed positive effects of wastewater irrigation that increased the abundance of earthworm communities, soil porosity, water retention and hydraulic conductivity.
\end{abstract}

Keywords: Wastewater irrigation, earthworm abundance, water retention, hydraulic conductivity, soil porosity

Résumé

La pratique de l’irrigation avec les eaux usées est une solution 
prometteuse pour atténuer le problème du stresse hydrique dans les régions semi-arides. Notre étude s’intéresse à l'impact de la pratique de l'irrigation avec les eaux usées sur l’abondance lombricienne sur la porosité des sols prairial des régions semi arides de l'est Algérien, ainsi que l’interaction entre les deux composantes sur le fonctionnement hydrique du sol. L'analyse des eaux à révélé une pollution organique et particulaire élevée cette dernière n’a aucune influence sur les propriétés physiques des sols. Nos résultats révèlent des effets favorables de l'irrigation avec des eaux usées sur la l'amélioration de l'abondance des communautés lombriciennes, la porosité, la rétention en eau et la conductivité hydraulique.

Mots clés : Eau usée, abondance lombricienne, rétention en eau, conductivité hydraulique, porosité

\section{Introduction}

Aujourd'hui, Avec les conditions climatiques des régions arides et semi arides, l'eau est devenue un enjeu incontestable de ce fait l'établissement d'un équilibre entre la demande croissante en eau et la disponibilité des ressources en eaux reste un grand défi. L'utilisation des eaux usées en agriculture à pour but de minimiser la surexploitation des eaux douces. Cette dernière est envisagée comme une solution prometteuse à large utilisation dans le monde afin de gérer le problème de stress hydrique.

Ce n'est pas une pratique qui appartient à un passé proche. Scott et al., (2004) et Buechler et al., (2006) indiquent que cette dernière est une pratique ancienne dans le monde entier.

Drechsel et al. (2011), indiquent que pendant des siècles, les agriculteurs chinois ont utilisé les excréments humains et animaux comme engrais et qu'avant l'introduction des technologies de traitement, dans plusieurs villes européenne et nord américaines, les eaux usées étaient évacuées vers les champs agricoles. Jiménez et Asano (2004), estiment que plus de 20 millions d'hectare de terres agricoles dans les pays en voie de développement sont irrigués avec les eaux usées. Dans la plupart de ces pays il n'existe pas d'installations de traitement des eaux usées et lorsqu'il y en a elles ne fonctionnent pas correctement (Quadir et al., 2007), ce qui peut engendrer des répercussions sur la santé et l'environnement. Les mêmes auteurs signalent que l'utilisation des eaux usées recyclées a subit une croissance au cours des dernières années dans plusieurs pays en Moyen orient, Afrique du nord, la méditerranée, au Etas unies, l'Amérique latine et l’Australie.

La réutilisation des eaux usées en agricultures présente un certain nombre d’avantages parallèlement aux inconvénients bien connus. Beaucoup de faveurs rendent l'utilisation de l'eau usées en agriculture une pratique 
prometteuse: (i) l'eau usée est une source non conventionnelle fiable et disponible toute l'année et leur volume tend à augmenter, contrairement aux précipitations et permet la réduction des prélèvements de l'eau souterraine, (ii) l'eau usée est riche en éléments nutritifs et matières organiques ce qui permet de réduire les apports d'engrais, Pekrun et al. (2003), signalent une augmentation des teneurs en azote et carbone organiques dans les cinq à dix premiers centimètres de sol irrigué à l'eau usée et la quantité élevée de matières organiques permet de conserver une minéralisation importante en irrigué à l'eau usée, (iii) cette pratique permet de réduire la pollution de l'eau de surface en évitant leur déversement sur les oueds.

En contrepartie la pratique de l'irrigation avec les eaux usées présente un certain nombre de problèmes: (i) les eaux usées utilisées pour l'irrigation peuvent contenir des différents contaminants d'ordre pathogènes : comme les bactéries, virus et les parasites et dans ce cas peuvent causer des risques sanitaires et environnementales (Oubrim et al., 2011), ainsi (ii) les effets sur le sol sont connus : l'arrivée des matières organiques et particulaire dans les sols peut affecter les propriétés physique et chimique de ces sols (Levy et al., 1999 ; Tarchitzky et al ., 1999 ; Mamdov et al., 2000). La dégradation des propriétés hydrauliques des sols a été étudiée par Levy et al., (1999). Tarchitzky et al. (1997), indiquent que les sels ajoutés lors de l'utilisation domestique et industrielle restent dans l'eau d'irrigation et peuvent éventuellement atteindre les sols et que plusieurs chercheurs ont déclaré la diminution de la conductivité hydraulique des sols aux quels l'eau usée est pratiquée. La gestion des sels dans les sols aux quels l'irrigation avec les eaux usées est appliquée est difficile car ces eaux contient une concentration élevée des sels et de composés organique et inorganique (Stevens et al., 2004). Les études de Buechler et Devi Gayathri (2006) indiquent une accumulation importante de $\mathrm{Cu}, \mathrm{Zn}$ et $\mathrm{P}$ dans les sols irrigués avec l'eau usée.

Dans le cadre de notre travail, nous nous sommes intéressés à l'étude de l'effet des eaux usées sur le fonctionnement du sol. Notre objectif est de comprendre l'impact de la pratique de l'irrigation à l'eau usée sur les deux composantes physique et biologique du sol ainsi que l'interaction entre les deux composantes sur le fonctionnement hydrique du sol.

\section{Matériel et méthodes}

Notre étude a eu lieu dans les prairies naturelles Sétifiennes au nord est de l’Algérie. Ces dernières sont irriguées par les eaux d'oued Boussellam qui représente le principal axe hydrographique de Sétif, avec une longueur de 159 km et un volume régularisable annuellement de près de $38 \mathrm{Hm}^{3}$.

Depuis les années soixante Boussellam est alimenté par les eaux usées de la ville de Sétif. Et de puis ce temps, parce qu’il présente une source 
permanente et gratuite d'eau et de fertilisants, cet Oued est utilisé pour l'irrigation de l'espace prairial ce qui rend, d'après Boudjenouia et al. (2006), les prairies naturelles plus fréquentes dans la région. Mais cette pratique, surtout que l’oued reçoit des eaux usées brutes, en plus des eaux de lavage et même des déchets solides de toute nature sont déversés dans l'Oued sans oublié les effluents agricoles, a des répercussions graves sur la santé et sur l'environnement.

Le climat de la région est continental semi- aride avec des étés chauds et secs et hivers rigoureux, les pluies sont insuffisantes et irrégulières et varient entre $251.7 \mathrm{~mm}$ et $614.73 \mathrm{~mm}$ (sur une période de 23ans (19922011) la moyenne est de $402.49 \mathrm{~mm}$ avec le cumul le plus faible $251.7 \mathrm{~mm}$, alors que en 2011 nous avons enregistré le cumul plus élevé 614.73mm) (tableau 1)

Tableau 1 : Variations interannuelles des précipitations (1992/2011) (ONM)

\begin{tabular}{|l|l|l|l|l|l|l|l|l|l|l|}
\hline Année & $\mathbf{1 9 9 2}$ & $\mathbf{1 9 9 3}$ & $\mathbf{1 9 9 4}$ & $\mathbf{1 9 9 5}$ & $\mathbf{1 9 9 6}$ & $\mathbf{1 9 9 7}$ & $\mathbf{1 9 9 8}$ & $\mathbf{1 9 9 9}$ & $\mathbf{2 0 0 0}$ & $\mathbf{2 0 0 1}$ \\
\hline $\mathbf{P P}(\mathrm{mm})$ & 467.62 & 386.89 & 254.06 & 418.59 & 421.70 & 352.57 & 445.08 & 384.50 & 331.00 & 251.7 \\
\hline Année & $\mathbf{2 0 0 2}$ & $\mathbf{2 0 0 3}$ & $\mathbf{2 0 0 4}$ & $\mathbf{2 0 0 5}$ & $\mathbf{2 0 0 6}$ & $\mathbf{2 0 0 7}$ & $\mathbf{2 0 0 8}$ & $\mathbf{2 0 0 9}$ & $\mathbf{2 0 1 0}$ & $\mathbf{2 0 1 1}$ \\
\hline $\mathbf{P P}(\mathrm{mm})$ & 371.5 & 590.10 & 454.91 & 373.8 & 354.83 & 411.20 & 418.34 & 403.10 & 422.67 & 614.73 \\
\hline
\end{tabular}

Les hautes plaines Sétifiennes se caractérisent par de grandes variations thermiques, on se référant au tableau 2 qui représente la répartition des températures mensuelles moyennes, maximale et minimale durant une période étalée sur 31 années (1981- 2011), on remarque que la température moyenne varie entre $5.48 \mathrm{C}^{\circ}$ au mois de janvier et $26.38 \mathrm{C}^{\circ}$ au mois de juillet. De même on constate que la température minimale la plus faible est enregistrée au mois de janvier $\left(1.35 \mathrm{C}^{\circ}\right)$, alors qu'une température maximale de $33.57 \mathrm{C}^{\circ} \mathrm{s}$ 'observe au mois de juillet.

Tableau 2 : Répartition des températures moyennes mensuelles durant la période (1981- 2011) (ONM)

\begin{tabular}{|c|c|c|c|c|c|c|c|c|c|c|c|c|}
\hline & Jan & Fev & Mar & Av & Mai & Juin & Juil & Aou & Sep & Oct & Nov & Déc \\
\hline T & 5.48 & 6.57 & 9.24 & 12.05 & 16.97 & 22.54 & 26.38 & 25.99 & 21.10 & 16.17 & 10.12 & 6.44 \\
\hline TM & 9.71 & 11.25 & 13.46 & 17.54 & 23.06 & 29.37 & 33.57 & 32.92 & 27.20 & 21.47 & 14.56 & 10.45 \\
\hline Tm & 1.35 & 1.93 & 4.19 & 6.51 & 10.81 & 15.68 & 19.16 & 19.11 & 15.13 & 11.02 & 5.76 & 2.48 \\
\hline
\end{tabular}

\section{Echantillonnage}

Pour apprécier l'impact de l'irrigation avec l'eau usée sur le sol des prairies nous avons choisis deux sites le premier site (S Urbain) ce sont des sols des prairies irrigués avec les eaux usées générées de la ville de Sétif et déversées directement dans l'oued Boussellem et le deuxième site ( $\mathrm{S}$ Agricole) où les sols des prairies reçoivent des eaux de l'oued Boussellem qui ont comme origine les eaux pluviale ainsi que les eaux de lixiviation des 
terres agricoles potentielles du nord de la ville de Sétif et nous l'avons considérer comme effluents agricoles (S Agricole). Dans chaque site nous avons réalisé trois répétitions pour l'ensemble des paramètres.

La caractérisation physico-chimique des eaux a été effectuée au cours de la saison pluviale sur des échantillons prélevés dans des bouteilles en polyéthylène de 1.5 litre de telle façon qu'il n'y est aucune bulle d'air et qu'il ne soit pas éjecte au cours du transport (Rodier, 1996) La mesure de température, $\mathrm{pH}, \mathrm{CE}$ et Oxygène dissous a été réalisée sur place. Les chlorures, nitrates, phosphates, les sulfates ainsi que l'ammonium calcium et magnésium sont analysées selon Rodier et al. (2009). La quantité des MES a été obtenue par centrifugation (Rejsek, 2002). La mesure de DBO5 à l'aide d'un DBO mètre et DCO par un DCO mètre. Le $\mathrm{Na}, \mathrm{K}, \mathrm{Fe}, \mathrm{Cu}, \mathrm{Zn}$ et Mn ont été analysés par spectrophotométrie à absorption atomique.

Pour l'étude de l'abondance lombricienne nous avons utilisé la méthode de Raw (1959), suivi d'un tri manuel sur le volume d'action.

La densité apparente a été mesurée sur le terrain avec des cylindres métalliques de $250 \mathrm{~cm}^{3}$ de volume. La porosité a été calculée à une densité réelle de $2.65 \mathrm{~g} / \mathrm{cm}^{3}$.

La conductivité hydraulique proche de la saturation k(h) est mesurée in situ à quatre potentiels de pression différents $(-1.5,-0.6,-0.3$ et - 0.06 $\mathrm{kPa}$ ) à l'aide d'un infiltromètre multi-disques à succion contrôlée TRIMS avec un diamètre moyen de $80 \mathrm{~mm}$.

La mesure de l'évolution de la teneur en eau des échantillons des deux traitements étudiés en fonction de la pression appliquée sur ces échantillons a été réalisée dans des presses de richards. Des cylindres ont été utilisés pour prélever les échantillons. La teneur en eau volumique a été mesurée pour des différents $\mathrm{pF}: 0.3,0.78,1.18,1.5,2,2.5$, et 4.2

Les résultats ont été soumis à une analyse statistique, il s'agit notamment d'une analyse en composantes principales (ACP) effectuée, en utilisant le logiciel statistique XLSTAT version 2015.5.01 23305 ;

\section{Résultats et discussion}

\section{Caractérisation physico-chimique des eaux}

L’appréciation de la qualité d'eau se fait d'après la détermination des paramètres de la caractérisation des eaux. Les caractéristiques physicochimiques des deux types d'eau sont regroupées dans la figure 1. L'examen de la composition physicochimique moyenne des eaux des deux sites montre quelles ont, pour la plupart des paramètres, des concentrations faibles qui ne dépassent guère la norme algérienne des eaux de surfaces et les normes de réutilisation des eaux usées en agriculture. Ceci est valable pour : $\mathrm{pH}$, température, $\mathrm{CE}, \mathrm{Ca}^{++}, \mathrm{Mg}^{++}, \mathrm{Na}^{+}, \mathrm{K}^{+}, \mathrm{NH}^{+}, \mathrm{NO}^{-}, \mathrm{SO}^{+}, \mathrm{PO}^{-}, \mathrm{Zn}$, et l’oxygène dissous. 
On constate une pollution intense pour la charge organique et particulaire, mais plus accentuée pour les eaux usées. Il s'agit des trois paramètres : DCO DBO5 et MES. En effet les charges moyennes exprimées en termes de DCO et DBO5 ont atteint des teneurs de l'ordre de $294 \mathrm{mg} / \mathrm{l}$ et $34 \mathrm{mg} / \mathrm{l}$ pour DCO et $235 \mathrm{mg} / \mathrm{l} 27 \mathrm{mg} / \mathrm{l}$ pour DBO5 respectivement pour les eaux usées et les effluents agricoles. Ces valeurs dépassent les valeurs guides fixées à $30 \mathrm{mg} / \mathrm{l}$ et $90 \mathrm{mg} / \mathrm{l}$ pour DCO et $7 \mathrm{mg} / \mathrm{l}$ et $30 \mathrm{mg} / \mathrm{l}$ pour DBO5 respectivement pour les normes algériennes des eaux de surfaces et des eaux usées destinées à la réutilisation en 'irrigation des sols agricoles. De même les valeurs très élevées sont enregistrées pour les matières en suspension (587 mg/l et $300 \mathrm{mg} / \mathrm{l}$ respectivement pour les eaux usées et les effluents agricoles).

Les teneurs en chlorures sont élevées pour les deux sites $(669 \mathrm{mg} / \mathrm{l}$ et $572 \mathrm{mg} / \mathrm{l}$ pour les eaux usées et les effluents agricoles respectivement).

\section{Abondance lombricienne \\ l'irrigation avec les eaux usées favorise l'abondance des communautés lombriciennes avec une valeur moyenne de 47 ind. $/ \mathrm{m}^{2}$, cette valeur représente le double du nombre total des vers dénombrés au niveau des sites irriguées avec les effluents agricoles (20 ind./ $\mathrm{m}^{2}$ ) (figure 2). Pour les deux modalités d'irrigations, les vers immatures dominent.}




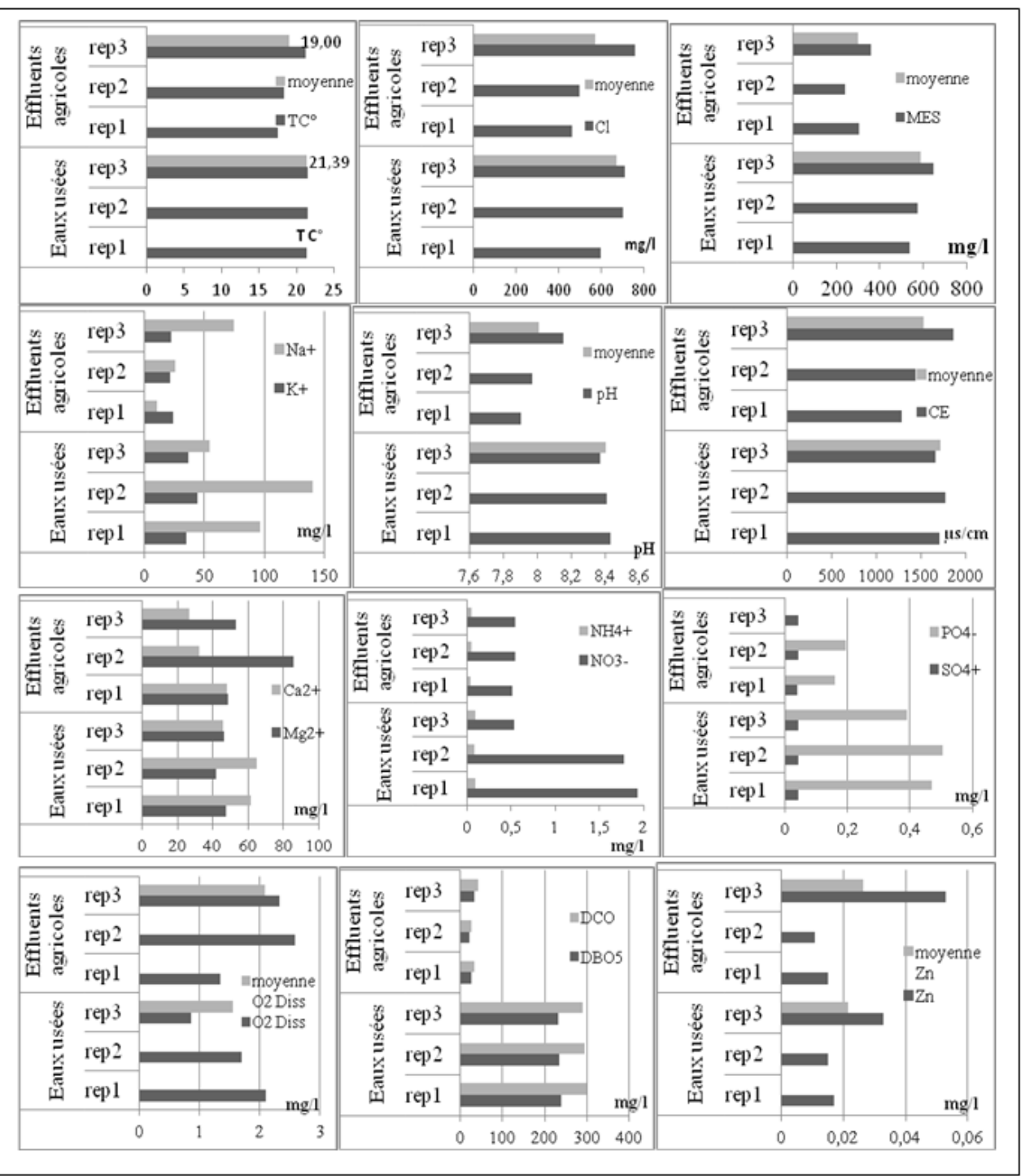

Figure 1. Résultats des analyses physico-chimiques des deux types d’eaux d’irrigation

L'étude de Bottinelli (2010), indique un nombre plus important de lombriciens sous apport de fumier de volaille par rapport a la fertilisation minérale. Anderson et al. (1983), indiquent qu'un apport de lisier ou fumier influence positivement les populations des vers de terre. La richesse des eaux usées en matières organiques et particulaire a favorisé l'abondance lombriciene dans les prairies.

Nos résultats évoquent que la qualité de l'eau d'irrigation influence sur l'abondance lombricienne totale. Avec un classement décroissant : eaux usées $<$ effluents agricoles. 


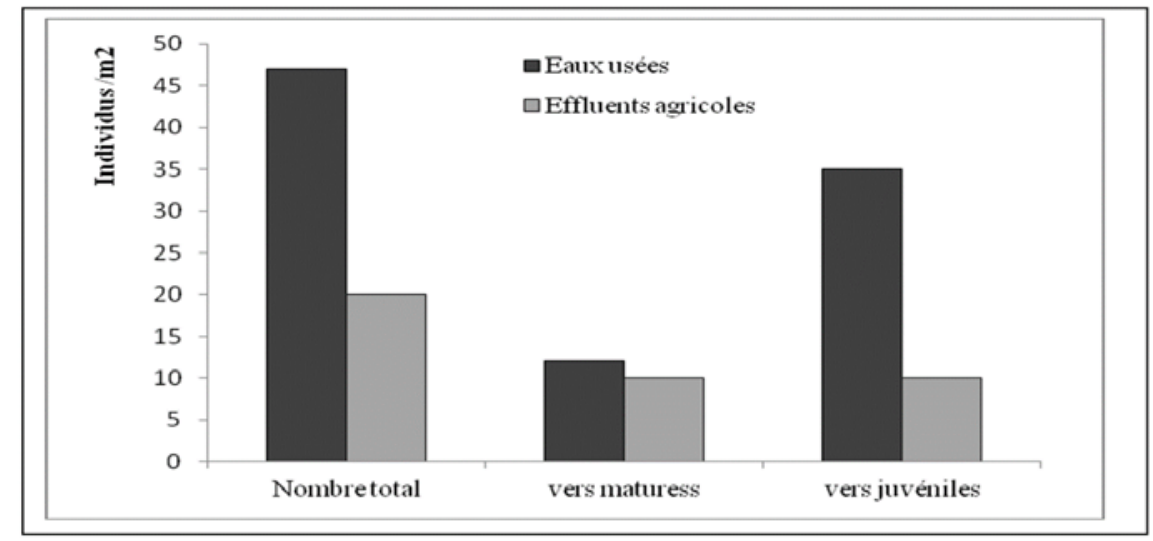

Figure 2. Abondance lombricienne dans les sols des prairies irriguées avec les deux modalités (eaux usées et effluents agricoles).

\section{Conductivité hydraulique}

Les valeurs moyennes de la conductivité hydraulique proche à saturation mesurées sur les sols irrigués avec les deux modalités sont rapportées dans la figure 3. Nous avons mesuré une forte conductivité hydraulique dans les sols irrigués à l'eau usée par rapport à celle mesuré dans les sols des prairies irrigués aux effluents agricoles, surtout pour les potentiels les plus proches de la saturation $\left(\mathrm{K}_{(-0.06)}=73.50 \mathrm{~mm} / \mathrm{h}\right.$ et $\quad \mathrm{K}_{(-0.3)}=$ $32.99 \mathrm{~mm} / \mathrm{h}$, pour les sols qui ont reçus l'eau usée comme substrat d'irrigation). Une légère augmentation a été observée pour les sols irrigués avec les effluents agricoles seulement pour le potentiel le plus négatif $(-1.5$ $\mathrm{kPa}$ ) par rapport à ceux irrigués à l'eau usée. (respectivement $4.94 \mathrm{~mm} / \mathrm{h}$ et $4.19 \mathrm{~mm} / \mathrm{h}$ ). Ces résultats sont en opposition avec ceux rapportés par plusieurs études qui témoignent la réduction de la conductivité hydraulique et l'infiltration dans les sols subissant l'irrigation avec les eaux usées (Bedbabis et al., 2014 ; Sou et et al., 2013 ; Li et al., 2010 ; Tarchouna et al., 2010 ; Gharaibeh et al., 2007 ; Viviani et Lovino, 2004).

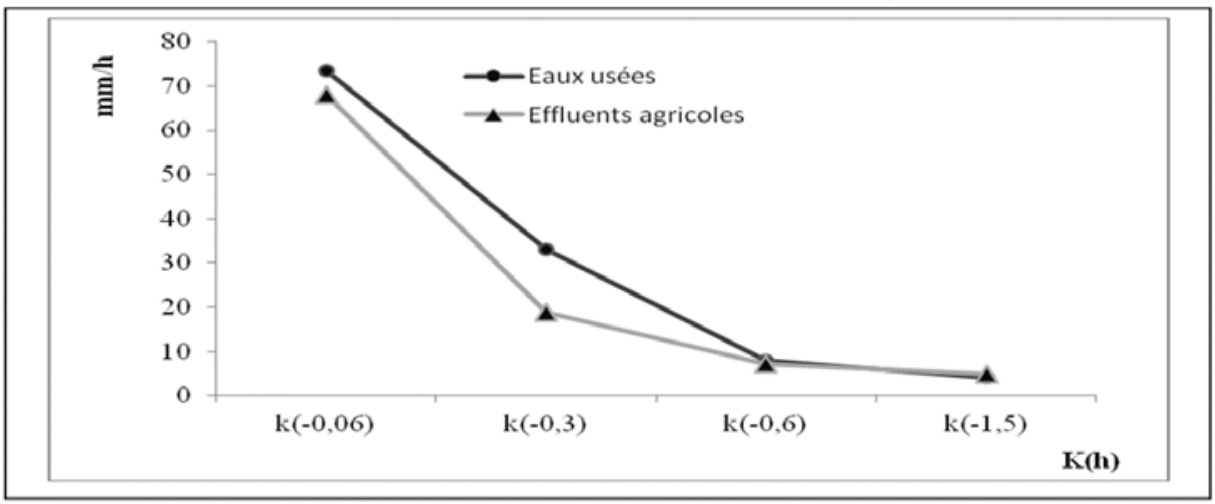

Figure 3. Conductivité hydrauliques des sols irrigués avec les eaux usées et les effluents agricoles. 


\section{Rétention en eau}

La figure 4 qui présente la relation entre l'humidité équivalente et le potentiel matriciel nous montre que quelque soit le potentiel appliqué, la courbe de rétention en eau des sols irrigués avec les eaux usées est supérieur que celle des sols irrigués avec les effluents urbains, donc retiennent plus d'eau. Les deux courbes se rapprochent seulement dans deux potentiels 0.6 $\mathrm{KPa}$ et $1584 \mathrm{KPa}$ qui correspond respectivement à $\mathrm{pF} 0.78$ et $\mathrm{pF} 4.2$.

Zupanc et Justin (2010), évoquent l'augmentation de la quantité d'eau disponible sous irrigation à l'eau usée, ce qui concorde avec nos résultats.

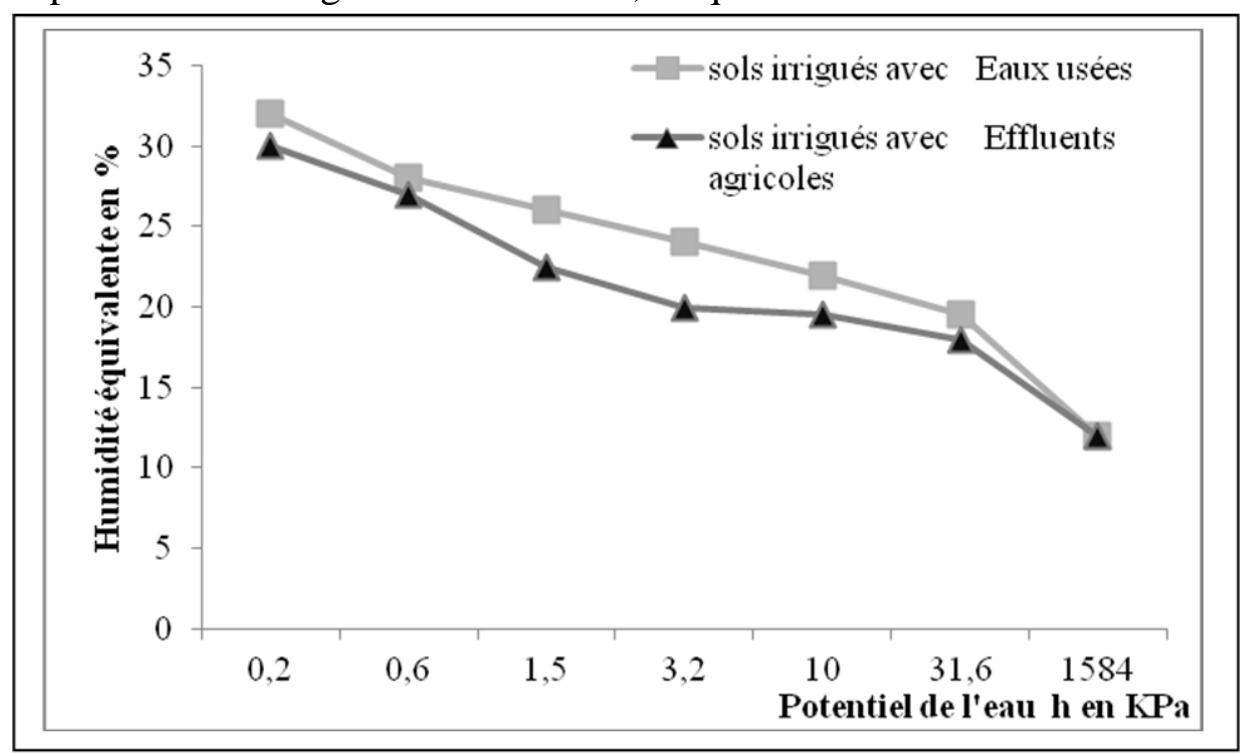

Figure 4. Relation entre le potentiel matriciels et la rétention en eau des sols irrigués avec les eaux usées et les effluents agricoles.

\section{La porosité}

La porosité totale moyenne calculée à partir des mesures de densité apparente, varie entre les deux modalités d'irrigation. La figure 5 révèle une variation du même ordre que l'abondance des lombrics où les sols des prairies irrigués avec les eaux usées sont les plus poreux $(\mathrm{P}=49.49 \%)$. Tandis-que les sols irrigués avec les effluents agricoles révèlent les valeurs de porosité les plus faibles. Ce qui indique que la qualité de l'eau d'irrigation influence sur la porosité avec le même ordre de son influence sur l'abondance des vers. Nos résultats corroborent avec d'autre études Tunc et Sahin (2015) ; Nadav et al. (2013) ; Mojiri (2011) ; Minhas et Samra, (2004). Alors que d'autres recherches indiquent des effets défavorables de l'irrigation avec les eaux usées sur la porosité des sols (Mollahoseini, 2013 ; Al Othman, 2009). 


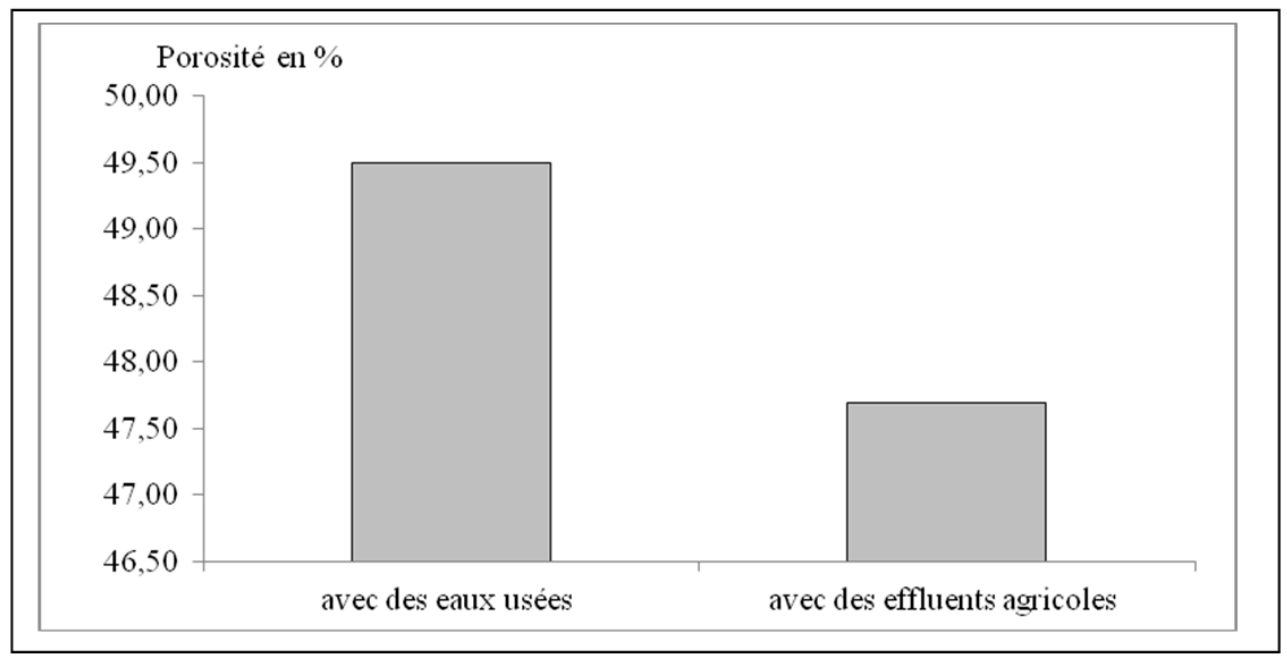

Figure 5. Porosité moyenne des sols irrigués avec les eaux usées et les effluents agricoles.

Nous avons réalisé une ACP pour représenter graphiquement la corrélation entre les paramètres biologiques (adulte, juvénile, total lombric) et physiques des sols dans les deux sites qui représentent les deux modalités d'irrigation.

La matrice de corrélation nous indique une corrélation positivement significative entre les lombrics immatures et la conductivité hydraulique aux potentiels proches à la saturation $\left(\mathrm{k}_{(0.06)} \mathrm{r}=0.89, \mathrm{k}_{(0.3)} \mathrm{r}=0.99, \mathrm{k}_{(0.6)} \mathrm{r}=0.95\right)$ ainsi avec la porosité $\mathrm{r}=0.97$ et les potentiels matriciels qui séparent les deux courbes de rétentions des sols irrigués avec les eaux usées et les effluents agricoles $(1.5 \mathrm{kPa} \mathrm{r}=0.89,3.2 \mathrm{kPa} \mathrm{r}=0.92, \quad 10 \mathrm{kPa} \mathrm{r}=0.87$ et $31.6 \mathrm{kPa} \mathrm{r}=$ 0.89). Cette corrélation obtenue entre lombrics et les autres paramètres suit celle des Allison (1973), où il indique que les galeries creusés par les vers de terre ont tendance à diminuer la densité apparente (donc augmenter la porosité) du sol et augmenter l'aération et le drainage.

La figure 6 illustre que la plus grande information est donnée par l'axe I (73.86\%) avec un cumule des deux axes de $86.56 \%$.

Plusieurs auteurs indiquent que les activités des vers de terre améliorent la structure du sol et augmentent l'infiltration (Syres et Springett, 1983 ; Edwards et Shipita, 1998). Cela concorde à notre travail où nous avons enregistré des corrélations significatives entre les lombrics et la conductivité hydraulique proche à la saturation et exactement ce sont les juvéniles qui agissent beaucoup plus. 


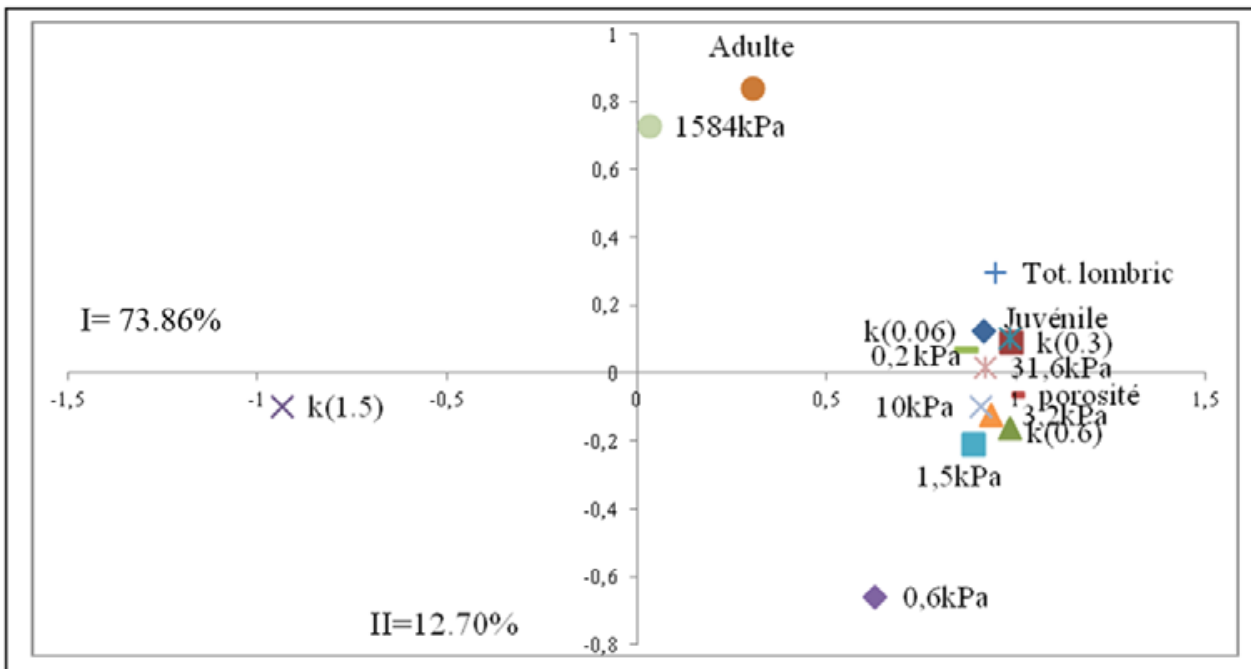

Figure 6. Représentation de la dispersion des individus dans le plans $1 / 22$ de l’ACP

Il ressort que nos résultats mettent en évidence que l'épandage des eaux usées améliore la porosité des sols, ce qui est en concordance avec les résultats de Minhas et Samra, (2004) qui indiquent une augmentation de la conductivité hydraulique et la porosité totale. Néanmoins nos résultats s’opposent à d'autres études. Molahoseini, (2014) ; Viviani et Lovino (2004); Sou (2009), qui signalent une diminution de la conductivité hydraulique qui peut être du au colmatage des pores.

\section{Conclusion}

Notre investigation illustre que l'irrigation avec l'eau usée provoque plus de porosité, une grande conductivité hydraulique et une bonne rétention en eau malgré la charge élevée de cette eau usée en matières organiques et particulaires cette dernière à favorisé l'abondance des communautés lombriciennes qui à influencé positivement sur les propriétés hydro physique des sols.

\section{References:}

Allison, F. E. 1973. Use of mulches. In F. E. Allison, ed. Soil organic matter and its role in crop production. Elsevier Scientific Publishing Company, New York.

Al Othman, A.A., 2009. Effect of treated domestic wastewater on physical and chemical characteristics of soils. Journal of applied sciences 9(5) pp.901908.

Anderson, J. M., Ineson, P. et Huish, S. A., 1983. Nitrogen and cation mobilization by soil fauna feeding on leaf litter and soil organic matter from deciduous woodlands. Soil Biol.Biochem. 15, 463-467. 
Bedbabis, S., Ben Rouina, B., BoukhrisM., Ferrara, G. 2014.Effect of irrigation with treated wastewater on soil chemical proprieties and infiltration rate. Journal of environmental management, pp. 133-450.

Bottinelli, N., 2010. Evolution de la structure et de la perméabilité d’un sol en contexte de non labour associé à l'apport d'effluent d'élevage : rôle de l'activité lombricienne. These doc. Agrocumpus Ouest Ecole nationale supérieure d'agronomie de rennes.152p.

Boudjenouia, A. Fleury, A. Tacherift, A. 2006. Le statut de l'espace agricole périurbain à Sétif (Algérie) : réserve foncière ou projet urbain. Cahiers Agricultures vol. 15, $\mathrm{n}^{\circ}$ 2, pp 221- 226.

Buechler, S., Gayathri Devi, M., 2006. Adaptations of wastewater-irrigated farming systems: A case study of Hyderabad, India. In van Veenhuizen, R. (Ed.). Cities farming for the future: Urban agriculture for green and productive Cities. Silang, Philippines: RUAF Foundation; IDRC; International Institute of Rural Reconstruction (IIRR) pp:267-273.

Buechler, S., Gayathri Devi, M., Ben, K., 2006. Wastewater use for urban and peri-urban agriculture. In van Veenhuizen, R. (Ed.) Cities farming for the future: Urban agriculture for green and productive cities. Silang, Philippines: RUAF Foundation; IDRC; International Institute of Rural Reconstruction (IIRR). Pp:244-260

Drechsel, P., Scott, C. A., Sally, L. R., Redwood, M. et Bahri, A., 2011. L’irrigation avec des eaux usées et la santé. Evaluer et atténuer les risques dans les pays à faible revenu. Bibliothèque et Archives nationales du Québec, 440p. ISBN 978-2-7605-3160-4

Edwards, W.M., Shipitalo, M.J., 1998. Consequences of earthworms in agricultural soils: aggregation and porosity. In: Edwards, C.A. (Ed.), Earthworm Ecology. CRC Press, Boca Raton, FL, pp. 147-161.

Gharaibeh, M.A., Eltaif, N.I., Al-Abdullah, B. 2007. Impact of field application of treated wastewater on hydraulic properties of vertisols. Water air soil pollution. 184, PP.347-353.

Jiménez, B., and Asano, T., 2004. “Acknowledge All Approaches: The Global Outlook on Reuse.” Water21 December 2004:32-37.

Levy, G.J., Rosenthal, A., Tarchitzky, J., Shainberg, I. and Chen, Y., 1999. Soil hydraulic conductivity changes caused by irrigation with reclaimed waste water. JEQ. Vol: $28 \mathrm{~N}^{\circ}$ 5. Pp 1658- 1664

Li, F. H., Yang, S. M. and Peng, C., 2010. Effects of domestic sewage water and ameliorant effectiveness on soil hydraulic conductivity. SSSAJ. Vol. 74 $\mathrm{N}^{\circ}$ 2, 461-468.

Mamedov, A.I., Shainberg, I. and Levy, G.J. 2000. Irrigation with effluent water: Effects of rainfall energy on soil infiltration. Soil Sci. Soc. Am. J., 64, 732-737.

Minhas, P.S. Samra, J.S., 2004. Wastewater use in peri-urban agriculture: 
impacts and opportunities. Tech. Bull. No. 2. Central Soil salinity Research Institute, Karnal 132001, India. 65 p.

Mollahoseini, H., 2013. Long term effects of municipal wastewater irrigation on some properties of a semiarid region soil of Iran. Int. J. Agron. Plant Product. Vol. 4 No. 5, pp. 1023-1028.

Molahoseini, H., 2014. Long term effects of municipal wastewater irrigation on some properties of a semi aride region soil of Iran. International Journal of Science Engineering and Technology V3 Issue 4, 444-449.

Mojiri, A. 2011. Effects of municipal wastewater on physical and chemical properties of saline soil. J. Biol. Environ. Sci., 5, pp. 71-76.

Nadav, I., Tarchitzky, J., Chen, Y., 2013.Water repellency induced by organic matter (OM) in treated wastewater (TWW) infiltration ponds and irrigation X. Jianming, W. Jianjun, H. Yan (Eds.), Functions of Natural Organic Matter in Changing Environment, Springer, Netherlands, pp. 883887.

Oubrim, N., Ennaji, M. M., Hajjami, K., Bennani, M., Hassar, M. et Cohen, N., 2011. Microbiological Impact of Treatment Lagoons on The economics of Water for Reuse in Agriculture a Case Study in Morocco (Settat and Soualem Regions). Cell. Mol. Biol. 57,

Pekrun, C., Kaul, H.P., Claupein, W., 2003. Soil tillage for sustainable nutrient management. In El Titi, A. (ed.), Soil tillage in agroecosystems, CRC Press, New-York (USA), pp. 83-113.

Quadir M., Shrma B. R., Bruggeman A. Choukr allah R. Karajeh F., 2007. Non conventional water resources and opportunities of water augmentation to achieve food security in water scare countries. Agricultural Water Management. Vol. 87 Issue 1, pp. 2-22

Raw, F., 1959. Estimating earthworm population by using formaline. Nature, 184 : 1661-1662. Raw, F., 1959. Estimating earthworm populations by using formalin. Nature 184, 1661-1662.

Rejsek, F. 2002. Analyse des eaux, Aspects réglementaires et techniques. Scérén TEC \& DOC. CRDP Aquitaine. 358 p.

Rodier, J., 1996. Analyse de l'eau : Eaux naturelles, Eaux résiduaires, Eau de mer. $8^{\text {ème }}$ Edition Dunod, Paris. 1383 p.

Rodier, J., Bernard, L., Nicole, M., et coll., 2009. L'analyse de l'eau. Eaux naturelles. Résiduaires. Eau de mer. 9ème édition. Dunod. Paris, 1526p.

Scott, C.A., Faruqui, N., and Raschid-Sally, L., 2004. Waste water use in irrigated Agriculture: Management challenges in developing Countries. In irrigated Agriculture Coordinating the livelihood and environmental realities. 10p.

Sou, Y.M., 2009. Recyclage des eaux usées en irrigation : potentiel fertilisant,_risques sanitaires et impacts sur la qualité des sols. Thèse DOC. É PF. Lausanne, 162 pages. 
Sou, Y.M., Mermoud, A., Yacouba, H., Boivin, P., 2013. Impact of irrigation with industrial treated wastewater on soil properties. Geoderma, 200-201, pp. 31-39.

Stevens, D., Unkovich, M., Kelly J., GouYing, G. 2004. Impacts on soil groundwater and surface water from continued irrigation of food and turf crops with water reclaimed from sewage. Australian Water Conservation and reuse Research Program.52p

Syers, J.K., Springett, J.A., 1983. Earthworm ecology in grassland soils. In: Satchell, J.E. (Ed.), Earthworm Ecology: From Darwin to Vermiculture. Chapman \& Hall, London, pp. 67-83.

Tarchitzky, J., Golobati, Y., Chen, Y. and Keren, R., 1997. Waste water effects on montmorillonite suspensions and hydraulic properties of sandy soils. Soil Science Society of America Journal (SSSAJ) vol: 63. N³. Pp: 554 $-560$.

Tarchitzky, J., Golobati, Y. Keren, R. and Chen, Y., 1999. Reclaimed wastewater effects on flocculation value of montmorillonite suspensions and hydraulic properties of a sandy soil. Soil Sci. Soc. Am. J. (63), 554-560.

Tarchouna, L. G., Merdy, P., Raynaud, M., Pfeifer, H.R. and Lucas, Y., 2010. Effects of long-term irrigation with treated wastewater. Part I: Evolution of soil physico-chemical properties, Applied Geochemistry, 25, pp 1703-1710. Tunc, T. \& Sahin, U. 2015. The changes in the physical and hydraulic properties ol a loamy soil under irrigation with simpler reclaimed wastewaters. Agricultural. Water Management, Vol.158, pp. 213-224.

Viviani, G. and Iovino, M., 2004. Wastewater Reuse Effects on Soil Hydraulic Conductivity, Journal of Irrigation and Drainage Engineering, Vol. 130, pp. 476-482.

Zupanc, V. \& Justin, M., 2010. Changes in soil characteristics during landfill leachate irrigation of Populus deltoides. Waste Management, 30, pp 21302136. 